\title{
The Moral of Hepatic Fibrosis: Don't Always Believe Noninvasive Fibrosis Measurements
}

\author{
Masaru Enomoto ${ }^{1} \cdot$ Norifumi Kawada $^{1}$
}

Published online: 1 January 2020

(c) Springer Science+Business Media, LLC, part of Springer Nature 2020

Chronic hepatitis C virus (HCV) infection affects approximately 71 million people worldwide, resulting in an estimated 399,000 deaths annually from HCV-related causes such as cirrhosis and hepatocellular carcinoma [1]. The development of direct-acting antiviral agents (DAA) has resulted in a substantial breakthrough in anti-HCV treatments. Although current interferon-free DAA treatments can achieve a sustained virologic response (SVR) in almost $100 \%$ of patients with chronic hepatitis C, SVR is merely a surrogate marker for virologic cure of $\mathrm{HCV}$ infection, since the ultimate goal of anti-HCV therapies is to prevent the development of cirrhosis and its morbid complications.

Liver fibrosis is a reversible wound healing reaction to persistent necro-inflammation that reflects a balance between extracellular matrix production and degradation. In the era of interferon-based therapy, a number of studies revealed that SVR in chronic hepatitis C patients resulted in long-term histological improvements in inflammation grade and fibrosis stage. Shiratori et al. [2] conducted a multicenter cohort study of 593 patients with chronic HCV infection (consisting of 487 treated and 106 untreated patients), who underwent a paired liver biopsy performed 3.7 years (median) apart in the interferon monotherapy era. The rate of fibrosis progression (in stages) was $-0.28 \pm 0.03$ unit/y (regression) in patients with SVR, $+0.02 \pm 0.02$ unit/y in patients without $S V R$, and $+0.10 \pm 0.02$ unit/y in untreated patients. Similarly in the peginterferon-ribavirin era, a metaanalysis showed that liver fibrosis was significantly reduced in patients with SVR and, to a lesser extent, in patients who relapsed but not non-responders [3].

In the DAA era, histological improvement after achieving SVR to DAA remains to be determined in patients with

Masaru Enomoto

enomoto-m@med.osaka-cu.ac.jp

1 Department of Hepatology, Osaka City University Graduate School of Medicine, 1-4-3, Asahimachi, Abeno, Osaka 545-8585, Japan chronic hepatitis $\mathrm{C}$. In contrast, an increasing number of studies have shown that DAA-induced SVR can improve noninvasive clinical variables, such as liver stiffness measurement (LSM), aspartate aminotransferase-to-platelet ratio index (APRI), and Fibrosis-4 (FIB-4) index. For example, many groups evaluated paired LSM using transient elastography before and after DAA therapy. A meta-analysis indicated a rapid regression in LSM during DAA therapy and a slower but steady regression after completion of treatment; in patients who achieved SVR, LSM decreased by $2.4 \mathrm{kPa}$ at the end of therapy, by $3.1 \mathrm{kPa} 1-6$ months after therapy, by $3.2 \mathrm{kPa} \mathrm{6-12} \mathrm{months} \mathrm{after} \mathrm{therapy,} \mathrm{and}$ by $4.1 \mathrm{kPa}>12$ months after therapy (median decrease $28.2 \%$; interquartile range 21.8-34.8) [4]. Nevertheless, it is well known that the results of LSM can be affected not only by fibrosis but also by inflammation in the liver. Furthermore, since APRI and FIB-4 indices including hepatic aminotransferases were originally proposed based on data obtained from chronic hepatitis $\mathrm{C}$ patients, their diagnostic accuracy has not been fully validated in healthy individuals or in patients who have achieved SVR. Thus, the rapid regression of LSM in the early days of treatment is primarily attributable to the regression of inflammation.

Liver biopsy, although invasive, has been considered the gold standard, at least in the past, for diagnosing chronic liver diseases. Liver biopsy is helpful for staging, grading inflammatory activity, and identifying comorbidities such as hepatic steatosis and iron overload. Of interest, Whitcomb et al. [5] reported that among 36 allograft liver biopsy specimens from patients who achieved SVR (to either interferon-based or interferon-free regimens) after receiving liver transplant, $69 \%$ still had histological features of active inflammation. Putra et al. [6] reported that among 25 patients who achieved SVR after receiving DAA treatment while on the liver transplantation waiting lists, there was a trend toward a higher degree of persistent inflammation, especially lytic necrosis/apoptosis and portal inflammation, in patients who achieved SVR in $<24$ weeks. We 
also observed that among less advanced chronic hepatitis C patients, approximately $18 \%$ of the 51 post-SVR biopsy specimens taken after DAA treatment showed clinically significant inflammation ( $\geq$ grade 2 ) even after excluding those with abnormal aminotransferase levels despite achieving a SVR [7]. In our analyses of 20 paired biopsy specimens, the overall grade of inflammatory activity and iron accumulation significantly regressed, whereas the stage of fibrosis did not, probably due to the short observation period. Yet, fibrous bundles/septa bridging between portal tracts became thin, possibly suggesting regression of liver fibrosis. Pan et al. [8] reported that among 15 patients with advanced fibrosis or cirrhosis (F3/F4) according to a pre-SVR liver biopsy who underwent a repeat biopsy post-SVR, only 4 patients showed improvement with F1-to-F2 fibrosis, whereas 11 patients still showed F3-to-F4 fibrosis. Nonetheless, morphometric analyses of the first 11 biopsied patients revealed that collagen content declined by an average of $46 \%$ over varying time intervals in 10 patients.

In this issue of Digestive Diseases and Sciences, Huang et al. [9] retrospectively analyzed paired liver biopsy specimens from patients with chronic HCV infection who attained SVR after receiving DAA; pre-SVR biopsy specimens were obtained at an average of 3 (range 1-6) months pre-treatment, and post-SVR biopsy specimens were obtained at an average of 6 (range 5-7) months post-treatment. Of the 40 patients with valid paired biopsy specimens, $33(83 \%)$ and $15(38 \%)$ exhibited short-term improvements in liver inflammation and fibrosis, respectively; the regression of inflammation was statistically significant $(P=0.000)$ although fibrosis was not $(P=0.547)$. Noninvasive measurements such as LSM, APRI, and FIB-4 significantly decreased after SVR and are predictive of fibrosis even after SVR; areas under the receiver operating characteristics curves for LSM, APRI, and FIB-4 were $0.86,0.86$, and 0.85 for prediction of post-SVR cirrhosis, respectively. The baseline LSM, APRI, and FIB-4 values were significantly higher in patients without regression in fibrosis post-SVR $(P=0.003-0.012)$.

This study is important since very few studies so far have evaluated the short-term histological changes after SVR has been achieved following DAA treatment. Nevertheless, it is not practical to histologically evaluate regression of liver fibrosis in a large number of patients who have achieved SVR; noninvasive clinical variables are important surrogates for liver biopsy in clinical practice. We have to keep in mind that the cutoff values for noninvasive measurements for predicting advanced fibrosis or cirrhosis decreased significantly after SVR, likely due to an improvement in hepatic inflammation. Although the American Gastroenterological Association guidelines recommended the cutoff of $<9.5 \mathrm{kPa}$ to exclude advanced liver fibrosis after SVR is achieved [10], this recommendation was supported by low-quality evidence according to the Grading of Recommendations Assessment,
Development, and Evaluation [GRADE] guidelines [11]. In the study by Huang et al. [9]; the optimal cutoff LSM values for cirrhosis before and after SVR were $6.8 \mathrm{kPa}$ and $5.3 \mathrm{kPa}$; the cutoff APRI values were 0.68 and 0.27 ; and the cutoff FIB-4 values were 1.15 and 1.17 , respectively. Notably, the cutoff values for cirrhosis were lower than those reported in previous studies. Although the reason for this discrepancy is unknown, the LSM results can be influenced by factors other than liver fibrosis, including inflammatory activity, hepatic congestion, extrahepatic cholestasis, obesity, and narrow intercostal space. Moreover, the authors noted that the small sample size might also have been contributory. Further studies with larger sample sizes are needed to define the optimal cutoff values for advanced fibrosis or cirrhosis in patients with normal aminotransferase values so as to not overestimate the regression in liver fibrosis after SVR is achieved by the noninvasive measurements.

Lastly, the present study did not include patients with decompensated, HCV-related cirrhosis in whom percutaneous liver biopsy was contraindicated based on the presence of ascites or coagulopathy due to thrombocytopenia or clotting factor deficiency. Although DAA treatment has recently been indicated even for decompensated patients, it remains controversial whether there is a 'point of no return,' defined as the degree of liver dysfunction at which HCV treatment no longer provides a clinical benefit. Huang et al. [9] reported that only $2 / 14$ (14\%) cirrhotic patients exhibited regression in liver fibrosis as seen in post-SVR biopsy, whereas the same was noted in 13/26 (50\%) patients without cirrhosis. Noninvasive measurements are also desired to identify patients with decompensated but reversible liver disease who are likely to benefit from DAA treatment.

\section{References}

1. World Health Organization. Hepatitis C. http://www.who.int/ mediacentre/factsheets/fs164/en/. Accessed 31 Dec 2019.

2. Shiratori $\mathrm{Y}$, Imazeki $\mathrm{F}$, Moriyama $\mathrm{M}$, et al. Histologic improvement of fibrosis in patients with hepatitis $\mathrm{C}$ who have sustained response to interferon therapy. Ann Intern Med. 2000;132:517-524.

3. Cammà C, Di Bona D, Schepis F, et al. Effect of peginterferon alfa-2a on liver histology in chronic hepatitis $\mathrm{C}$ : a meta-analysis of individual patient data. Hepatology. 2004;39:333-342.

4. Singh S, Facciorusso A, Loomba R, Falck-Ytter YT. Magnitude and kinetics of decrease in liver stiffness after antiviral therapy in patients With chronic hepatitis C: a systematic review and metaanalysis. Clin Gastroenterol Hepatol. 2018;16:27-38.e4.

5. Whitcomb E, Choi WT, Jerome KR, et al. Biopsy specimens from allograft liver contain histologic features of hepatitis $\mathrm{C}$ virus infection after virus eradication. Clin Gastroenterol Hepatol. 2017;15:1279-1285.

6. Putra J, Schiano TD, Fiel MI. Histological assessment of the liver explant in transplanted hepatitis $\mathrm{C}$ virus patients achieving 
sustained virological response with direct-acting antiviral agents. Histopathology. 2018;72:990-996.

7. Enomoto M, Ikura Y, Tamori A, et al. Short-term histological evaluations after achieving a sustained virologic response to direct-acting antiviral treatment for chronic hepatitis C. United Eur Gastroenterol J. 2018;6:1391-1400.

8. Pan JJ, Bao F, Du E, Skillin C, et al. Morphometry confirms fibrosis regression from sustained virologic response to direct-acting antivirals for hepatitis C. Hepatol Commun. 2018;2:1320-1330.

9. Huang R, Rao H, Yang M, et al. Noninvasive measurements predict liver fibrosis well in hepatitis $\mathrm{C}$ virus patients after directacting antiviral therapy. Dig Dis Sci. (Epub ahead of print). https ://doi.org/10.1007/s10620-019-05886-y.

10. Lim JK, Flamm SL, Singh S, Falck-Ytter YT, Clinical Guidelines Committee of the American Gastroenterological Association.
American Gastroenterological Association Institute Guideline on the Role of Elastography in the Evaluation of Liver Fibrosis. Gastroenterology. 2017;152:1536-1543.

11. Guyatt GH, Oxman AD, Vist GE, et al. An emerging consensus on rating quality of evidence and strength of recommendations. BMJ. 2008;336:924-926.

Publisher's Note Springer Nature remains neutral with regard to jurisdictional claims in published maps and institutional affiliations. 